\title{
Maladie de Crohn diagnostiquée par les ulcérations buccales : à propos d'un cas
}

\section{Blein E, Curien R. (UFR d'Odontologie, Nancy)}

La maladie de Crohn est une affection inflammatoire chronique siégeant généralement au niveau de l'iléon même si elle peut affecter n'importe quel segment de l'appareil digestif (de la cavité buccale jusqu'à l'anus). Sa cause est probablement immunitaire due à un antigène bactérien ou viral. Elle est fréquemment retrouvée soit chez l'adulte de moins de 30 ans, soit chez l'enfant et indépendant du sexe.

Une patiente de 25 ans se plaint à la consultation du service d'odontologie d'ulcérations buccales non douloureuses présentes depuis plusieurs semaines. II s'agit de fissures linéaires profondes, au fond du cul-de-sac vestibulaire inférieur. De pseudos polypes plus ou moins aplatis de consistance molle sont présents au sein de ces fissures et en périphérie. A l'interrogatoire, la patiente rapporte avoir eu une fissure anale l'année précédente avec abcès ano-périnéal traité par Bristopen ${ }^{\circledR}$.

Un bilan sanguin comprenant NFS, VS, CRP a été réalisé. Cette dernière est augmentée à 12mg/L.

La biopsie réalisée le jour de la consultation révèle de nombreux petits granulomes épithélioïdes associés à quelques cellules plurinucléées de type Langhans. Cette caractéristique et l'antécédent de fissure anale avec abcès ano-périnéal sont tout à fait compatible avec le diagnostic d'une maladie de Crohn.

La patiente est donc adressée au service d’hépato-gastro-entérologie.

Une IRM montre la présence de deux trajets fistuleux actifs sans abcès. L'entéro-IRM est négative. Une endoscopie objective la présence d'ulcérations œsophagiennes pouvant s'intégrer dans la maladie de Crohn. Le diagnostic de maladie de Crohn avec localisation buccale et ano-périnéal est donc retenu.

Le traitement comporte une bithérapie de fluoroquinolone et de métronidazole ainsi qu'une chirurgie (drainage en séton) puis un traitement par anti-TNF alpha.

Les lésions buccales de la maladie de Crohn peuvent précéder ou accompagner les lésions intestinales ou même rester isolées. Elles sont le plus souvent caractéristiques : ce sont des granulomes giganto-épithélioïdes faits de cellules épithélioïdes avec parfois des cellules de Langhans isolées au sein d'un infiltrat lymphoplasmocytaire.

Toute ulcération atypique de la muqueuse buccale sans étiologie évidente doit faire évoquer un diagnostic de maladie inflammatoire chronique intestinale. Dans tous les cas, seule la biopsie permettra un diagnostic de certitude.

BLEIN Eleonore

eleonoreblein@gmail.com

This is an Open Access article distributed under the terms of the Creative Commons Attribution License 2.0, which permits unrestricted use, distribution, and reproduction in any medium, provided the original work is properly cited. 